by using routine data from an executive check-up program of an international company and to examine the relationship between ALI and work-related surrogate health parameters.

Methods Datasets from 307 examinations of 151 executives $(19$ ㅇ $132 \precsim)$ were available. Each participant attended at least one check-up examination between 2003 and 2015. The mean of age was 43.6 (SD $\pm 6.6,31-64$ y). We developed four different ALI. Thyreotropin was used as a proxy variable for a primary mediator [MacLean, 1994]. For each ALI the association to the Work Ability Index (WAI) and the category of sick leave days (SLD) was examined by using generalised linear mixed models. Zero inflation was considered for SLD. All analyses were conducted with R 3.3.3 or SPSS 23 .

Results ALI 1 showed a significant negative association with the WAI $(B=-0.680, S E=0.266, p=0.049)$. The results for ALI 2 had a similar trend $(B=-0.355, S E=0.201, p=0.081)$. The higher the ALI the lower the workability was rated. After adjustment for zero inflation ALI 3 and 4 showed a positive association with SLD, that is, there is a significant difference between the category of no SLD and any SLD.

Conclusion This study led to first hints that biomarkers form a secondary prevention program are useful to calculate a meaningful ALI. This ALI could be used as a marker in workplace health promotion. Further studies with a longitudinal approach and a broader range of occupations are recommended.

\section{EXPLORATION OF DIABETES \& CARDIOVASCULAR RISKS FACTORS AMONG OIL SECTOR WORKERS IN KUWAIT: 2013 PME \& COMPARISONS TO THE POPULATION}

Mohammad Hasan. Kuwait Oil Company, Ahmadi, Kuwait

\subsection{6/oemed-2018-ICOHabstracts.497}

Background High levels of diabetes and associated cardiovascular risk factors have been reported in Kuwait. While there is limited research on prevalence and risk factors in the general population, the associations of diabetes with cardiovascular (CVD) disease risk factors among oil industry employees have not yet been extensively explored. This aim of this study was to establish prevalence of diabetes and associations between diabetes and CVD risk factors broken down by gender and ethnicity.

Methods A cross-sectional study of 7000 employees of a major oil company (corresponding to $94 \%$ of the total population of employees) was conducted. $18.1 \%$ were female and $26.8 \%$ were non-Kuwaiti nationals. Clinical (blood samples) and nonclinical data (e.g. weight, height, and medically diagnosed chronic conditions) were gathered upon their visit to a clinic and medical laboratory.

Results Prevalence of obesity (using BMI) in the sample was $33.3 \%$. 35.8\% were physically active. The prevalence of diabetes was $15.6 \%$, of dyslipidemia $47.9 \%$ and of hypertension $14.8 \%$. Advancing age ( $\geq 40$ years), male gender, obesity, physical activity, high triglycerides, hypertension and were significantly associated with increased risk of diabetes in a multivariate analysis. Ethnicity also played a role.

Conclusion This study shows that among the cardiovascular disease risk factors reported by oil sector employees in Kuwait, apart from age; hypertension is of particular importance as a predictor of diabetes, especially for women. Health behaviours (e.g. smoking and physical activity) did not show expected or consistent multivariate associations with diabetes, across gender and nationality.

\section{COLLECTION OF COUNTRY-WIDE AND CULTURE- SPECIFIC HM DATA TO IMPROVE EFFECTIVENESS OF HEALTH PROGRAMS}

${ }^{1,2}$ Chaitanya Gulvady. 'Siemens Ltd, Mumbai, India; '2Indian Assocaition of Occupational Health

\subsection{6/oemed-2018-ICOHabstracts.498}

Introduction A comprehensive health program initiated in a corporate enabled the setting up/upgrading of health infrastructure, collection and analysis of data and launching of wellness programs in the workplace, to improve the physical and psychosocial health, the lifestyle and work life balance of employees.

Methods An elaborate Healthy@Siemens Label program was launched in 2015, which involved.

Management consent, Health Committees and As Is Analysis of health services. Measures were then planned to upgrade health infrastructure, improve collection and analysis of data and introduce interventional health measures. The following data collection was enabled.

- Sickness absenteeism

- Hospitalisation data

- Counselling data

- Body Biometrics

- Annual Health Checkups

- Morbidity Prevalence.

Result The data collected demonstrated the physical and psychosocial morbidity in employee population and health profile of employees. Measures such as the interventional Fit4Life program for Obesity, Diabetes, Blood pressure, HealthyLeadership@Siemens program for Managers, monthly Health promotion and awareness programs, vaccination drives for infective diseases, Introduction of a health portal and monthly reminders were the interventions that ensued.

Discussion Collection of comprehensive health data and introduction of interventional need based, sustained, targeted programs in workplaces leads to reduction in morbidity, in sickness absenteeism and improvement in body parameters of employees. There was early referral of employees with psychosocial issues by Managers for counselling. These measures have resulted in an improvement in the wellbeing of employees with resultant improved work lif balance, increase in performance and productivity and increased ownership culture in the organisation.

\section{INFLUENCING WORKERS' WILLINGNESS TO SEEK HELP AFTER A WORKERS' HEALTH SURVEILLANCE}

Karen Nieuwenhuijsen*, Carel T Hulshof, Judith K Sluiter. Academic Medical Center, Coronel Institute of Occupational Health, APH research institute, Amsterdam, NL

\subsection{6/oemed-2018-ICOHabstracts.499}

Introduction In Workers' Health Surveillance, workers are presented with their results of preventive tests. How the test results should be presented in this context in order to influence help-seeking behaviour, e.g. visiting a health provider, is 
not known. The aim of this study is to examine the influence of presentation of the results of a preventive medical examination on willingness to seek help for workrelated fatigue or being overweight.

Methods A factorial design experiment with counterbalancing was conducted by presenting $\mathrm{n}=82$ workers with vignettes including eight scenarios with hypothetical preventive tests results. The results were presented by stating either:

i. a 'high score' only (Neutral label),

ii. a 'high score, followed by a statement emphasising the risk of a current disorder' (Current label), or

iii. a 'high score, followed by statement emphasising the risk of this situation progressing into a health condition in the future' (Progress label).

Participants rated the willingness to seek help on a VAS scale (0-not at all willing to 100 -very willing) as if these were their own results. Differences between pairs of scenarios were tested with paired-sample t-tests.

Result Compared to the presentation with neutral labels, participants reported more willingness to seek help in both the scenarios with current vs neutral pairs (46, SD 27.1, vs 37, SD 27.1; p<0.000), and the progress vs neutral pairs $(47$, SD 27.6 vs 36 , SD $26.0 ; p<0.000)$. No statistically significant differences were observed between scenarios about work-related fatigue and being overweight.

Discussion Workers are more inclined to seek help if the risk is explicitly presented in the results.

Our experimental design allowed us to compare various conditions, but we could not use actual test results. Testing whether workers react differently to results reflecting their own health rather than vignettes remains a challenge for future research.

\section{PERSONAL PROTECTIVE EQUIPMENT FOR PREVENTING HIGHLY INFECTIOUS DISEASES DUE TO EXPOSURE TO CONTAMINATED BODY FLUIDS IN HEALTHCARE STAFF}

\begin{abstract}
${ }^{1}$ Jos Verbeek* ${ }^{*}{ }^{1}$ Sharea ljaz, ${ }^{1}$ Christina Tikka, ${ }^{1} J a n i$ Ruotsalainen* ${ }^{2}$ Erja Mäkelä, ${ }^{3}$ Kaisa Neuvonen, ${ }^{4}$ Michael Edmond, ${ }^{5}$ Riitta Sauni, ${ }^{6}$ FSelcen Kilinc Balci, ${ }^{1}$ Raluca Mihalache. ${ }^{1}$ Cochrane Work, Finnish Institute of Occupational Health, Kuopio, Finland; ${ }^{2}$ Finnish Institute of Occupational Health, Helsinki, Finland; ${ }^{3}$ Cochrane Work, Finnish Institute of Occupational Health, Helsinki, Finland; ${ }^{4}$ University of lowa Hospitals and Clinics, lowa City, IA, USA; ${ }^{5}$ Finnish Institute of Occupational Health, Tampere, Finland; ${ }^{6}$ National Personal Protective Technology Laboratory (NPPTL), National Institute for Occupational Safety and Health (NIOSH), Centres for Disease Control and Prevention (CDC), Pittsburgh, PA, USA
\end{abstract}

\subsection{6/oemed-2018-ICOHabstracts.500}

Introduction In epidemics of highly infectious diseases, such as Ebola Virus Disease (EVD) or SARS, healthcare workers $(\mathrm{HCW})$ are at much greater risk of infection than the general population, due to their contact with patients' contaminated body fluids. Personal protective equipment (PPE) can reduce the risk. It is unclear which type of PPE protects best, what is the best way to remove PPE, and how to make sure HCWs use PPE as instructed.

Methods We systematically searched for and included all eligible controlled studies that compared the effect of types or components of PPE in HCWs exposed to highly infectious diseases with serious consequences, such as EVD and SARS, on the risk of infection, contamination, or noncompliance with protocols. We also included studies that compared the effect of various ways of donning or removing PPE, and the effects of various types of training in PPE use on the same outcomes.

Result We found very low quality evidence that more breathable types of PPE may not lead to more contamination, but may have greater user satisfaction. We also found very low quality evidence that double gloving and CDC doffing guidance appear to decrease the risk of contamination and that more active training in PPE use may reduce PPE and doffing errors more than passive training. However, the data all come from single studies with high risk of bias and we are uncertain about the estimates of effects.

Discussion We need simulation studies, preferably using a nonpathogenic virus, to find out which type and combination of PPE protects best, and what is the best way to remove PPE. We also need RCTs of one type of training versus another to find out long term effects. HCWs exposed to highly infectious diseases should have their use of PPE registered.

\section{HOW AND WHEN ARE NONRANDOMISED STUDIES INCLUDED IN COCHRANE SYSTEMATIC REVIEWS - AN OVERVIEW OF CURRENT PRACTICE}

Sharea ljaz, Jos Verbeek, Christina Tikka, Jani Ruotsalainen*. Cochrane Work, Finnish Institute of Occupational Health, Kuopio, Finland

\subsection{6/oemed-2018-ICOHabstracts.501}

Introduction It is generally felt that randomised studies are difficult to conduct in the work-environment. Then again, nonrandomised studies (NRS) are considered to provide less reliable evidence for intervention effects. However, these are included in Cochrane reviews, despite discouragement. There has been no evaluation of when and how these designs are used. Therefore we conducted an overview of current practice.

Methods We included all Cochrane reviews that considered NRS. We conducted study screening and data extraction in duplicate.

Result Of the included 202 reviews, 114 (56\%) did not cite a reason for including NRS. In reviews that do cite a reason, the reasons were divided into two major categories: NRS were included because randomised trials (RCTs) are wanted ( $\mathrm{n}=81,92 \%)$ but not feasible, lacking, or insufficient alone, or because RCTs are not needed $(n=7,8 \%)$.

Review authors included a range of study designs with controlled before after studies being the most common.

Most interventions evaluated in Cochrane reviews incorporating NRS were non-pharmaceutical and the settings nonmedical.

For risk of bias assessment, most review authors (38\%) used Cochrane Effective Practice and Organisation of Care (EPOC) Group's checklists while others used a variety of checklists and self-constructed tools.

Discussion Most Cochrane reviews do not justify including NRS. Where they do, the majority is not in line with Cochrane recommendations. Risk of bias assessment varies across reviews and needs improvement. We provide an algorithm for when it is useful to include NRS in systematic reviews. If interventions are implemented at the group level as is often the case in occupational health studies, it is useful to also include NRS. 